\title{
Checking and Reasoning about Semantic Web through Alloy
}

\author{
Jin Song Dong, Jing Sun, and Hai Wang \\ School of Computing, \\ National University of Singapore, \\ dongjs, sunjing, wangh@comp.nus.edu.sg
}

\begin{abstract}
Semantic Web (SW), commonly regarded as the next generation of the Web, is an emerging vision of the new Web from the Knowledge Representation and the Web communities. The Formal Methods community can also play an important role to contribute to SW development. Reasoning and consistency checking can be useful at many stages during the design, maintenance and deployment of SW ontology. However the existing reasoning and consistency checking tools for SW are primitive. We believe that formal techniques and tools, such as Alloy, can provide automatic reasoning and consistency checking services for SW. In this paper, we firstly construct semantic models for the SW language (DAML+OIL) in Alloy, and these models form the semantic domain for interpreting DAML+OIL in Alloy. Then we develop the translation techniques and tools which can automatically map the SW ontology into the DAML+OIL semantic domain in Alloy. Furthermore, with the assistance of Alloy Analyzer (AA) we demonstrate that the consistency of the SW ontology can be checked automatically and different kinds of reasoning tasks can be supported.
\end{abstract}

keywords: Semantic Web, Alloy

\section{Introduction}

In recent years, researchers have begun to explore the potential of associating web content with explicit meaning so that the web content becomes more machine-readable and intelligent agents can retrieve and manipulate pertinent information readily. The Semantic Web (SW) [1] proposed by W3C is one of the most promising and accepted approaches. It has been regarded as the next generation of the Web. SW not only emerges from the Knowledge Representation and the Web Communities, but also brings the two communities closer together. We believe in the SW development process, there is a role for formal techniques and tools to play and make important contributions.

In the development of Semantic Web there is a pivotal role for ontology, since it provides a representation of a shared conceptualization of a particular domain that can be communicated between people and applications. Reasoning can be useful at many stages during the design, maintenance and deployment of 
ontology. Because autonomous software agents may perform their reasoning and come to conclusions without human supervision, it is essential that the shared ontology is consistent. However, since the Semantic Web technology is still in the early stage, the reasoning and consistency checking tools are very primitive.

The software modeling language Alloy [9] is suitable for specifying structural properties of software. SW is a well suited application domain for Alloy because relationships between web resources are the focus points in SW and Alloy is a first order declarative language based on relations. Furthermore, Alloy specifications can be analyzed automatically using the Alloy Analyzer (AA) 10]. Given a finite scope for a specification, AA translates it into a propositional formula and uses SAT solving technology to generate instances that satisfy the properties expressed in the specification. We believe that if the semantics of the SW languages can be encoded into Alloy, then Alloy can be used to provide automatic reasoning and consistency checking services for SW. Various reasoning tasks can be supported effectively by AA.

The remainder of the paper is organized as follows. Section 2 briefly introduces the Semantic Web and Alloy. In section 3 semantic domain and functions for the DARPA Agent Markup Language (DAML+OIL) 14 constructs are defined in Alloy. Section 4 presents the transformation from DAML+OIL documents to an Alloy program. In section 5 different reasoning tasks are demonstrated. Section 6 concludes the paper.

\section{Semantic Web and Alloy Overview}

\subsection{Semantic Web Overview}

The Semantic Web is a vision for a new kind of Web with enhanced functionality which will require semantic-based representation and processing of Web information. W3C has proposed a series of technologies that can be applied to achieve this vision. The Semantic Web extends the current Web by giving the web content a well-defined meaning, better enabling computers and people to work in cooperation. XML is aimed at delivering data to systems that can understand and interpret the information. XML is focused on the syntax (defined by the XML schema or DTD) of a document and it provides essentially a mechanism to declare and use simple data structures. However there is no way for a program to actually understand the knowledge contained in the XML documents.

Resource Description Framework (RDF) [11] is a foundation for processing metadata; it provides interoperability between applications that exchange machine-understandable information on the Web. RDF uses XML to exchange descriptions of Web resources and emphasizes facilities to enable automated processing. The RDF descriptions provide a simple ontology system to support the exchange of knowledge and semantic information on the Web. RDF Schema [2] provides the basic vocabulary to describe RDF documents. RDF Schema can be used to define properties and types of the web resources. Similar to XML Schema which gives specific constraints on the structure of an XML document, 
Table 1. DAML+OIL constructs (partial)

\begin{tabular}{||l|l||}
\hline DAML+OIL constructs & Description \\
\hline$D A M L \_c l a s s$ & classes \\
\hline$D A M L \_p r o p e r t y$ & properties \\
\hline$D A M L \_s u b c l a s s[C]$ & subclasses of C \\
\hline$D A M L \_s u b p r o p e r t y[P]$ & sub properties of P \\
\hline instanceof $[C]$ & instances of the DAML+OIL class C \\
\hline
\end{tabular}

RDF Schema provides information about the interpretation of the RDF statements. The DARPA Agent Markup Language (DAML) [14] is an AI-inspired description logic-based language for describing taxonomic information. DAML currently combines Ontology Interchange Language (OIL) [3] and features from other ontology systems. It is now called DAML+OIL and contains richer modelling primitives than RDF. The DAML+OIL language builds on top of XML and $\operatorname{RDF}(\mathrm{S})$ to provide a language with both a well-defined semantics and a set of language constructs including classes, subclasses and properties with domains and ranges, for describing a Web domain. DAML+OIL can further express restriction on membership in classes and restrictions on certain domains and ranges values.

Semantic Web is highly distributed, and different parties may have different understanding of the same concept. Ideally, the program must have a way to discover the common meanings from the different understandings. It is central to another important concept in Semantic Web service - ontology. The ontology for a Semantic Web service is a document or file that formally defines the relations among terms. The most typical kind of ontology for the Web has taxonomy and a set of inference rules. Ontology can enhance the functioning of the Web in many ways, and RDFS and DAML+OIL supply the language to define the ontology.

We summarize some essential DAML+OIL constructs in Table 1.

\subsection{Alloy Overview}

Alloy [9] is a structural modelling language based on first-order logic, for expressing complex structural constraints and behavior. Alloy treats relations as first class citizens and uses relational composition as a powerful operator to combine various structured entities. The essential constructs of Alloy are as follows:

Signature: A signature (sig) paragraph introduces a basic type and a collection of relation (called field) in it along with the types of the fields and constraints on their value. A signature may inherit fields and constraints from another signature.

Function: A function (fun) captures behaviour constraints. It is a parameterized formula that can be "applied" elsewhere,

Fact: Fact (fact) constrains the relations and objects. A fact is a formula that takes no arguments and need not to be invoked explicitly; it is always true. 
Assertion: An assertion (assert) specifies an intended property. It is a formula whose correctness needs to be checked, assuming the facts in the model.

The Alloy Analyzer (AA) is a tool for analyzing models written in Alloy. Given a formula and a scope - a bound on the number of atoms in the universe AA determines whether there exists a model of the formula that uses no more atoms than the scope permits, and if so, return it. It supports two kinds of automatic analysis: simulation, in which the consistency of an invariant or operation is demonstrated by generating a state or transition, and checking, in which a consequence of the specification is tested by attempting to generate a counterexample.

\section{DAML+OIL Semantic Encoding}

DAML+OIL has a well-defined semantics which has been described in a set of axioms [7]. In this section based on the semantics of DAML+OIL, we define the semantic functions for some important DAML+OIL primitives in Alloy. The complete DAML+OIL semantic encoding can be found in the appendix.

\subsection{Basic Concepts}

The semantic models for DAML+OIL are encoded in the module DAMLOIL. Users only need to import this module to reason DAML+OIL ontology in Alloy.

module DAMLOIL

All the things described in Semantic web context are called resources. A basic type Resource is defined as:

sig Resource \{\}

All other concepts defined later are extended from the Resource. Property, which is a kind of Resource itself, relates Resource to Resource.

\section{disj sig Property extends Resource}

\{sub_val: Resource $\rightarrow$ Resource\}

Each Property has a relation sub_val from set <Property, Resource, Resource> with type <Resource, Resource, Resource> (since in Alloy subsignature does not introduce a new type). This relation can be regarded as a RDF statement, i.e., a triple of the form

<property(or predicate), subject, value(or object)>.

The class corresponds to the generic concept of type or category of resource. Each Class maps a set of resources via the relation instances, which contains all the instance resources. The keyword disj is used to indicate the Class and Property are disjoint.

disj sig Class extends Resource \{instances: set Resource\} 
The DAML+OIL also allows the use of XML Schema datatypes to describe (or define) part of the datatype domain. However there are no predefined types in Alloy, so we treat Datatype as a special Class, which contains all the possible datatype values in the instances relation.

disj sig Datatype extends Class \{\}

\subsection{Class Elements}

The subClass0f is a relation between classes. The instances in a subclass are also in the superclasses. A parameterized formula (a function in Alloy) is used to represent this concept.

fun subClass0f (csup, csub: Class)

\{csub.instances in csup.instances\}

The disjointWith is a relation between classes. It asserts that there are no instances common with each other.

fun disjointWith (c1, c2: Class) \{no c1.instances \& c2.instances\}

\subsection{Property Restrictions}

A toClass function states that all instances of the class $c 1$ have the values of property $P$ all belonging to the class c2.

fun toClass ( $p$ : Property, c1: Class, c2: Class)

\{all $\mathrm{r} 1, \mathrm{r} 2$ : Resource $\mid \mathrm{r} 1$ in $\mathrm{c} 1$.instances $\Leftrightarrow \mathrm{r} 2$ in $\mathrm{r} 1$. (p.sub_val) $\Rightarrow$

$r 2$ in $c 2$.instances\}

A hasValue function states that all instances of the class c1 have the values of property $P$ as resource $r$. The $r$ could be an individual object or a datatype value.

fun hasValue (p: Property, $c 1$ : Class, $r$ : Resource)

\{all $r 1$ : Resource | $r 1$ in $c 1$.instances $\Rightarrow r 1 .\left(p . s_{0} \_v a l\right)=r$ \}

A cardinality function states that all instances of the class $c 1$ have exactly $\mathrm{N}$ distinct values for the property $\mathrm{P}$. The new version of Alloy supports some integer operations.

fun cardinality ( $\mathrm{p}$ : Property, $\mathrm{c} 1$ : Class, $\mathrm{N}$ : Int)

\{all $\mathrm{r} 1$ : Resourcel $\mathrm{r} 1$ in $\mathrm{c} 1$.instances $\Leftrightarrow \# \mathrm{r} 1 .\left(\mathrm{p} . \mathrm{sub}_{-} \mathrm{val}\right.$ ) $=$ int $\left.\mathrm{N}\right\}$

\subsection{Boolean Combination of Class Expressions}

The intersection0f function defines a relation between a class $c 1$ and a list of classes clist. The List is defined in the Alloy library. The class c1 consists of exactly all the objects that are common to all class expressions from the list clist. 


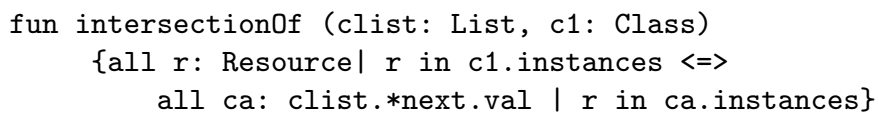

The union0f function defines a relation between a class $c 1$ and a list of classes clist. The class c1 consists of exactly all the objects that belong to at least one of the class expressions from the list clist. It is analogous to logical disjunction;

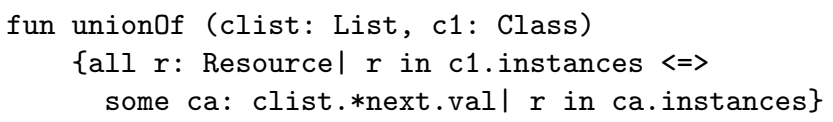

\subsection{Property Elements}

The subPropertyOf function states that psub is a subproperty of the property psup. This means that every pair (subject,value) that is in psup is in the psub. fun subPropertyOf (psup, psub: Property) \{psub.sub_val in psup.sub_val\}

The domain function asserts that the property $\mathrm{P}$ only applies to instances of the class $\mathrm{c}$.

fun domain (p: Property, c: Class) \{(p.sub_val).Resource inc.instances\}

The inverse0f function shows two properties are inverse.

fun inverse0f (p1, p2: Property) \{p1.sub_val = (p2.sub_val)\}

\section{DAML+OIL to Alloy Transformation}

In the previous section we defined the semantic model for the DAML+OIL constructs, so that analyzing DAML+OIL ontology in Alloy can be easily and effectively achieved. We also constructed a XSLT [15] stylesheet for the automatic transformation from DAML+OIL file to into Alloy program. 1

A set of transformation rules transforming from DAML+OIL ontology to Alloy program are developed in the following presentation.

\subsection{DAML+OIL Class Transformation}

$$
\frac{C \in D A M L_{-} \text {class }}{\text { static disj sig } C \text { extends Class }\{\}}
$$

A DAML_class C will be transferred into a scalar C, constrained to be an elements of the signature Class.

\footnotetext{
${ }^{1}$ The details of the XSLT program and other information on this project can be found at:

http://nt-appn.comp.nus.edu.sg/fm/alloy/
} 


\subsection{DAML+OIL Property Transformation}

$\frac{P \in D A M L_{-} \text {property }}{\text { static disj sig } P \text { extends Property }\{\}}$

A DAML_property $\mathrm{p}$ will be transferred into a scalar $\mathrm{P}$, constrained to be an elements of the signature Property.

\subsection{Instance Transformation}

$x \in$ instancesof $[Y]$
static disj sig $x$ extends Resource \{\}
fact $\{x$ in Y.instances $\}$

A DAML instance $\mathrm{x}$ of class $\mathrm{Y}$ will be transferred into a scalar $\mathrm{x}$, constrained to be an element of the signature Resource. $\mathrm{x}$ is a subset of $\mathrm{Y}$. instances.

\subsection{Other Transformation}

Other DAML+OIL constructs can be easily transferred into the Alloy function we defined in the previous section. For example the following rule shows how to transfer the DAML+OIL subclass relation into Alloy code.

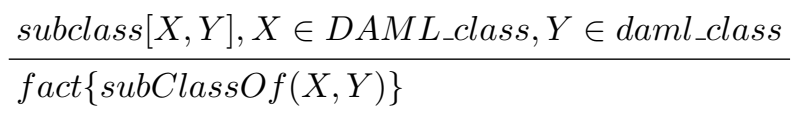

\subsection{Case Study}

A classical DAML+OIL ontology, "animal relation" is used to illustrate how the transformation and analysis could be achieved. The following DAML+OIL ontology defines two class animal and plant which are disjoint. The eats and eaten_by are two properties, which are inverse to each other. The domain of eats is animal. The carnivore is a subclass of animal which can only eat animals.

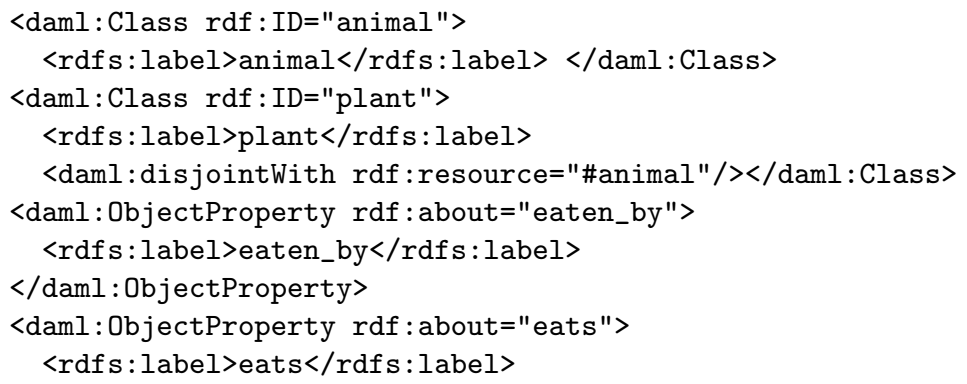




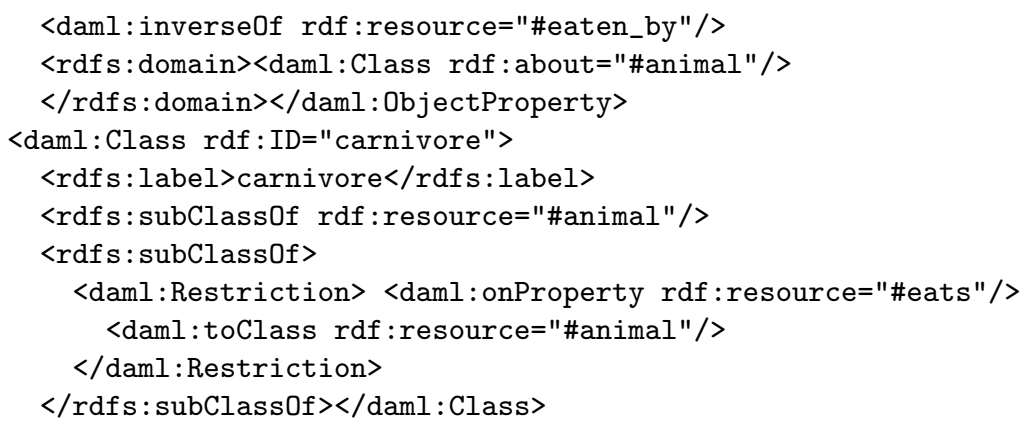

This DAML+OIL ontology will be transferred into Alloy as follow, module animal

We can check the consistency of the DAML+OIL ontology and do some reasoning readily.

\section{Analysing DAML+OIL Ontology}

Reasoning is one of the key tasks for the semantic web. It can be useful at many stages during the design, maintenance and deployment of ontology.

There are two different levels of checking and reasoning, the conceptual level and the instance level. At the conceptual level, we can reason about class properties and subclass relationships. At the instance level, we can do the membership checking (instantiation) and instance property reasoning. The DAML+OIL reasoning tool, i.e. FaCT [8], can only provide conceptual level reasoning, while AA can perform both. The FaCt system originally is designed to be a terminological classifer (TBox) which concerns only about the concepts, roles and attributes, not instances. The semantic web reasoner based on the FaCT, like OILED, does not support instance level reasoning well. 


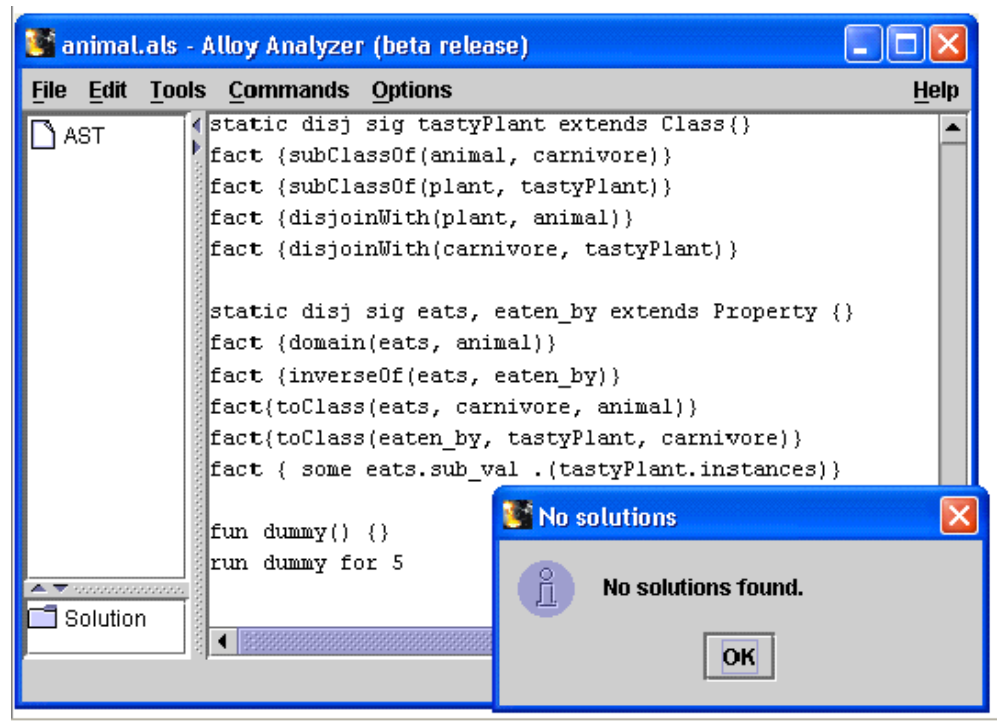

Fig. 1. Inconsistence example

\subsection{Class Property Checking}

It is essential that the ontology shared among autonomous software agents is conceptually consistent. Reasoning with inconsistent ontology may lead to erroneous conclusions. In this section we give some examples of inconsistent ontology that can arise in ontology development, and demonstrate how these inconsistencies can be detected by the Alloy analyzer. For example, we define another class tastyPlant which is a subclass of plant and eaten by the carnivore. There is an inconsistency since by the ontology definition carnivores can only eat animals. Animals and plants are disjoint.

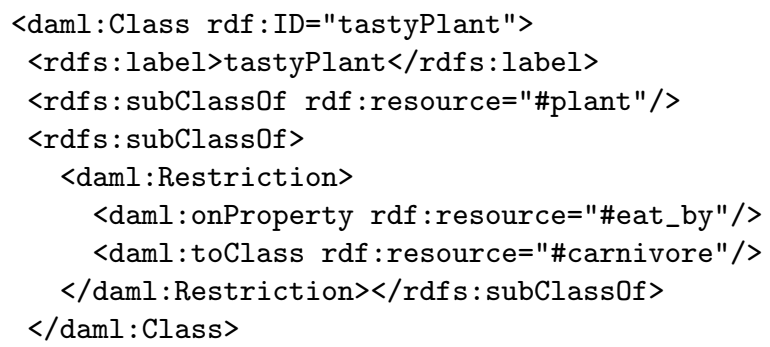

We transform the ontology into an Alloy program, add some facts to remove the trivial models (like everything type is empty set) and load the program into the Alloy Analyzer. The Alloy Analyzer will automatically check the consistency. We conclude that there is an inconsistency in the animal ontology since Alloy can not find any solutions satisfying all facts within the scope (Figure 1). Note that when Alloy can not find a solution, it may also be due to the scope being too small. By picking a large enough scope, "no solution found' is very likely to mean that an inconsistency has occurred. 
Let us take another example. Suppose we define that the polyphagic_animal eats at least two kind of things i.e polyphagic_animal objects have at least two distinct values for the property eats. There is also one kind of animal called picky_animal which only eats one other kind of animal. The ontology will be defined as follows:

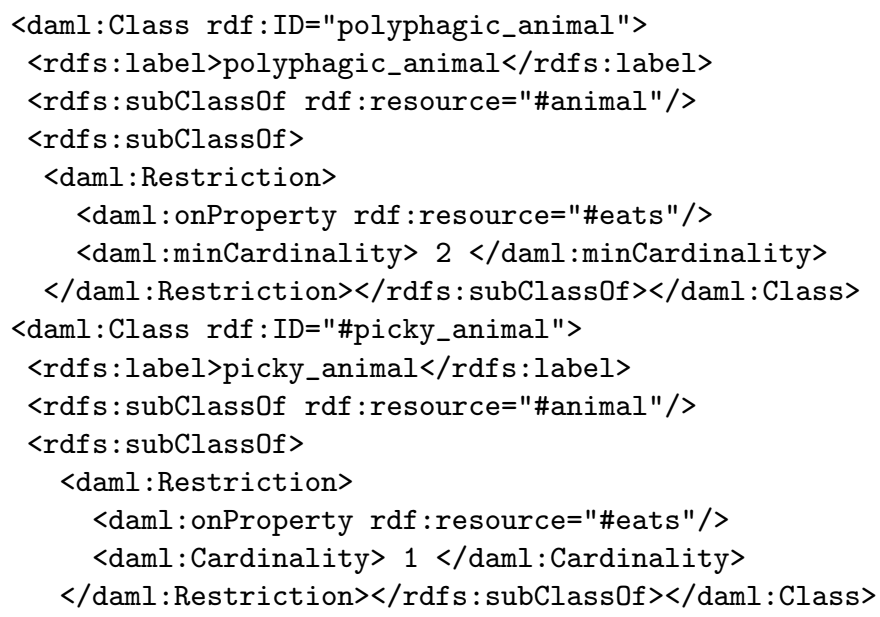

From the above ontology we can infer that the picky_animal is not a kind of polyphagic_animal, otherwise it would be an inconsistency that AA can easily pick up.

\subsection{Subsumption Reasoning}

The task of subsumption reasoning is to infer a DAML+OIL class is the subclass of another DAML+OIL class. We use the relationship between the fish, shark and dolphin as a example to demonstrate this kind of reasoning task. In the animal ontology a property breathe_by is defined. A fish class is a subclass of the animal which breathe_by the gill. Since the purpose of this paper is to demonstrate ideas, we keep the ontology simple. In reality there are some animals such as frogs and toads, which can respire by use of gills when they are young and by lungs when they reach adult stage. Also we do not consider the animals which respire by use of the pharyngeal lining or skin, like newborn Julia Creek dunnarts.

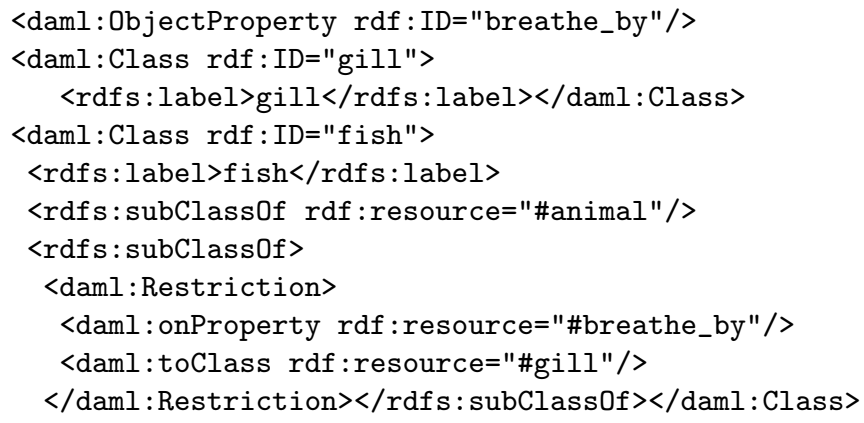




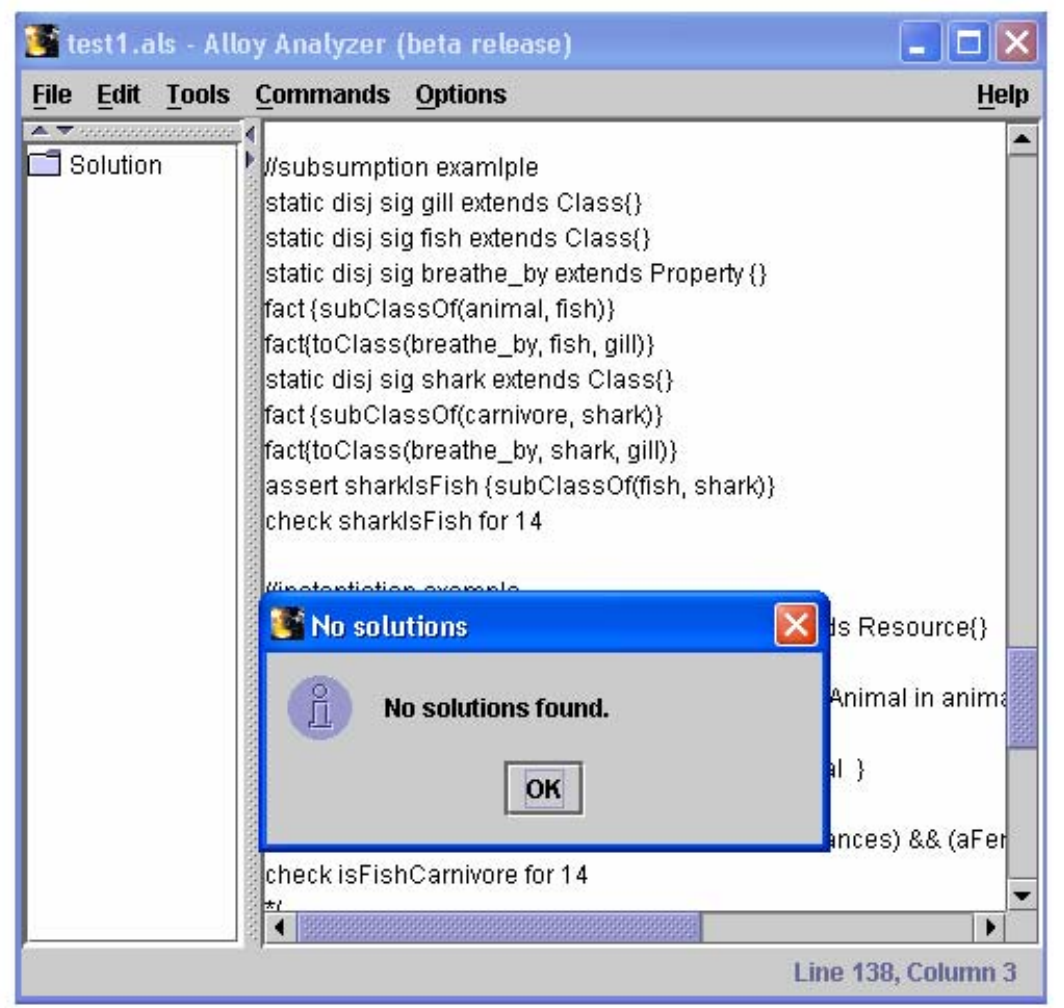

Fig. 2. Subsumption example

We also define a class shark, a subclass of carnivore which breathe by the gill.

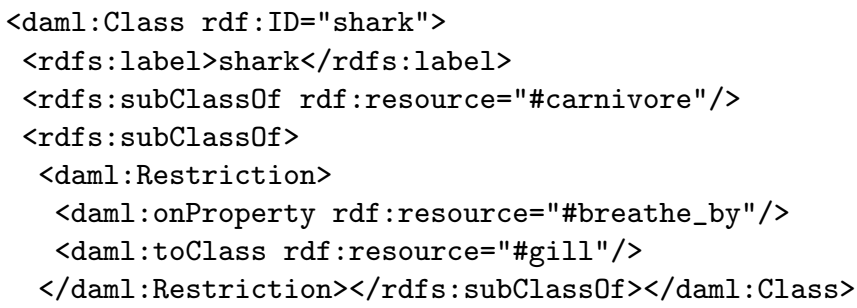

Several of the classes were upgraded to being defined when their definitions constituted both necessary and sufficient conditions for class membership, e.g., a animal is a fish if and only if it breathes by the gill. Additional subclass relationships can be inferred i.e. the shark is also a subclass of $f$ ish. We transfer this ontology into an Alloy program and make an assertion that the shark is the subclass of fish. The Alloy analyzer will check the correctness of this assertion automatically (Figure 2). The Alloy Analyzer checks whether an assertion holds by trying to find a counterexample. Note that "no solution" means no 


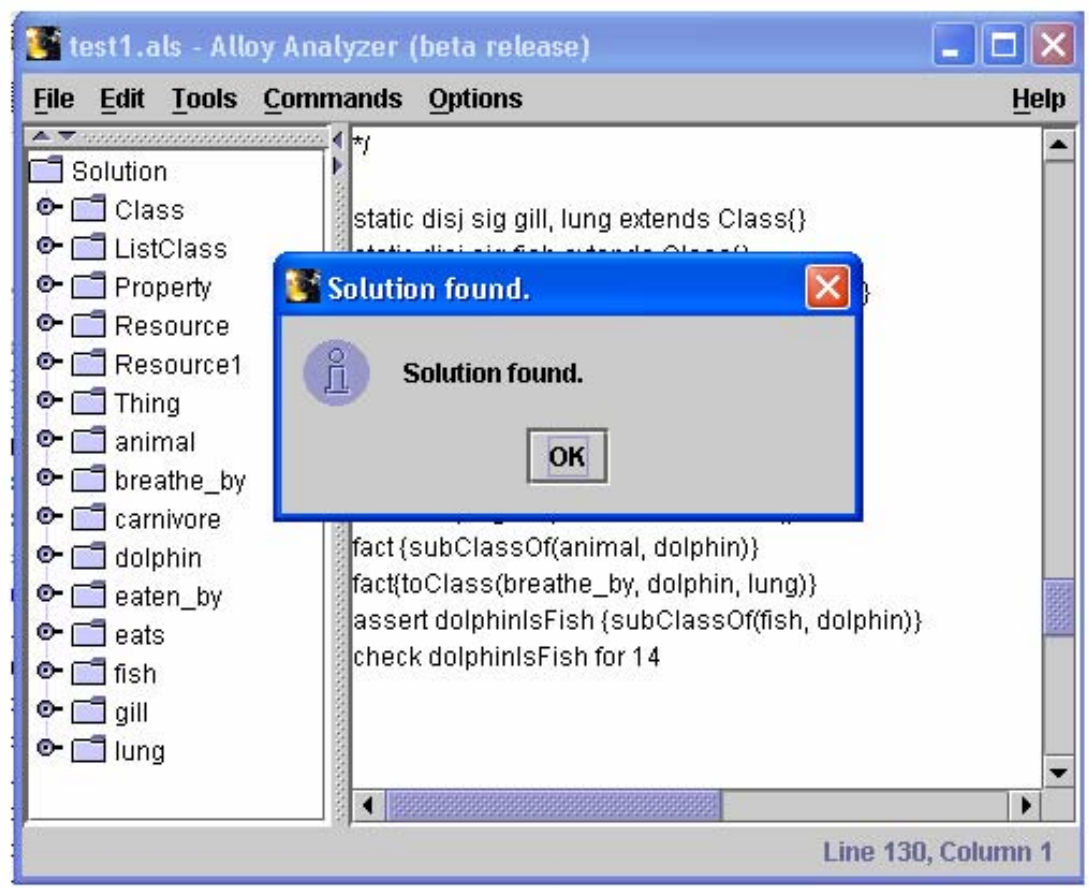

Fig. 3. Dolphin is not a fish

counterexample found, in this case, it indicates that the assertion is sound. To make it more interesting, we define classes dolphin and lung. The Dolphin is a kind of animal which breathe by lungs. The classes gill and lung are disjoint. Furthermore the breathe_by is a unique property.

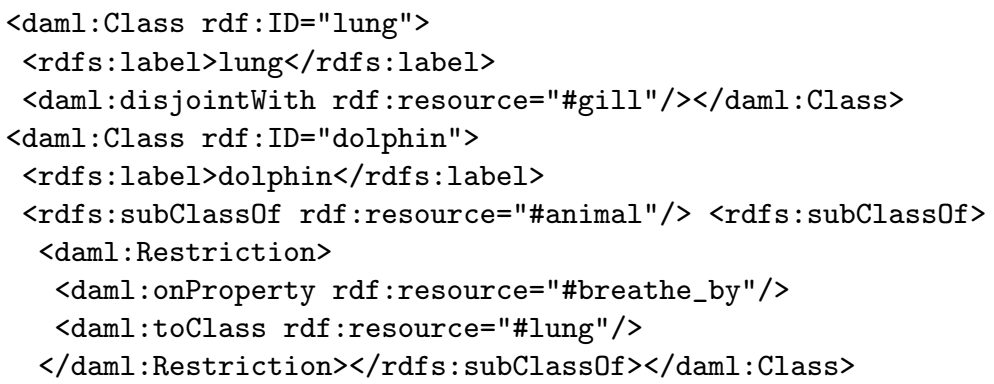

Suppose we make an assertion that the dolphin is a kind of $\mathrm{fish}$, the Alloy Analyzer will refute it since some counterexample was found (Figure 3). If we add that dolphin is a fish as a fact in the module, the AA will conclude that an inconsistency has arisen. 


\subsection{Instantiation}

Instance level reasoning is one of the main contributions for reasoning over DAML+OIL ontology using Alloy. Currently some successful DAML+OIL reasoners like $\mathrm{FaCT}$ are designed for description logics (DL) T-box reasoning, which lacks support for instances. In Alloy every expression denotes relations. The scalars will be represented by singleton unary relations - that is, relations with one column and one row. The instance level reasoning can be supported readily in Alloy.

Instantiation is a reasoning task which tries to check if an individual is an instance of a class. For example, we define two resources aFeralAnimal and aMeekAminal as the instances of class animal. aGill is an instance of class gill. aFeralAnimal eats aMeekAnimal and breathes by aGill. People may want to check if aFeralAnimal is a carnivore and a fish.

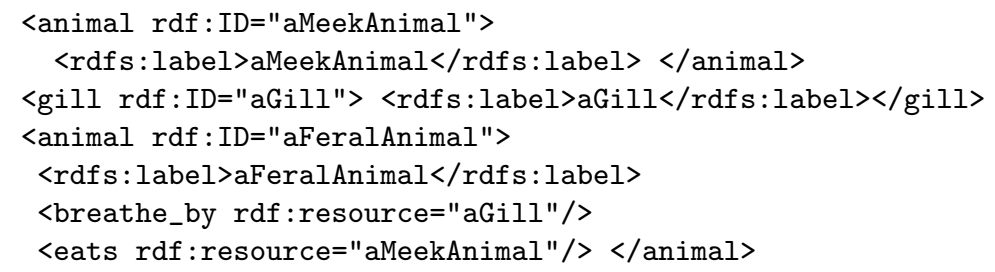

We transfer the ontology into an Alloy program and make an assertion as following:

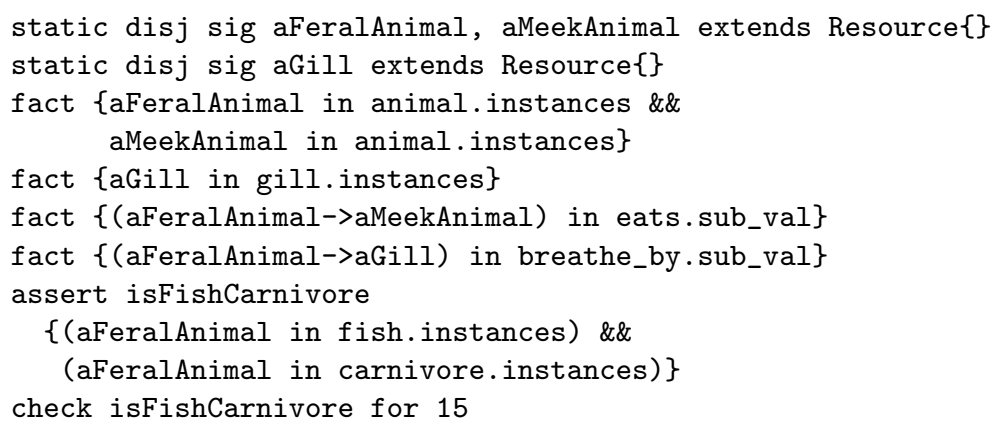

AA concludes that this assertion is correct.

\subsection{Instance Property Reasoning}

Instance property reasoning (often regarded as knowledge querying) is important in Semantic Web applications. Since one of the promising strengths of Semantic Web technology is that it gives the agents the capability to do more accurate and more meaningful searches. The agent can answer some questions for which the answer is not explicitly stored in the knowledge base.

For example, the emerge_early and emerge_later are two properties, which are inverse to each other. Animal A emerged early then B if the species of A 
emerges earlier than the species of $B$ on the earth. emerge_early is transitive. Three animal instances firstDinosaur, firstApe and firstHuman are defined. firstDinosaur emerge_early firstApe and firstApe emerge_early firstHuman. One possible question people may ask is that whether firstHuman is emerge_later firstDinosaur. With the assistance of Alloy reasoner, such questions can be answered.

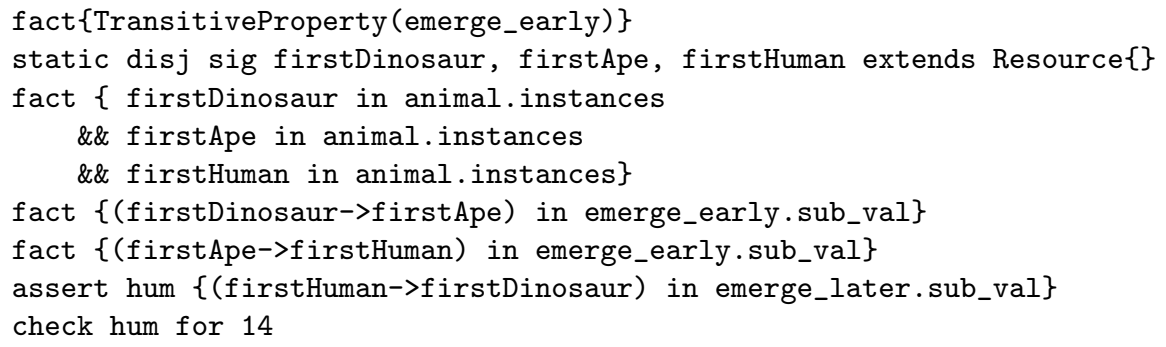

AA concludes that this assertion is correct.

\section{Related Work and Conclusion}

The main contribution of this paper is that it develops the semantic models for DAML+OIL language constructs in Alloy and the systematic transformation rules and (XSLT) program which can translate DAML+OIL ontology to Alloy automatically. With the assistance of Alloy Analyzer (AA), we also demonstrated that the consistency of the SW ontology can be checked automatically and different kinds of reasoning tasks can be supported. Alloy is chosen over other modeling techniques because

- Alloy is based on relations, where relations between web resources are the focus issues in SW.

- Alloy has an impressive automatic tool support.

We believe SW is a new novel application domain for Alloy. Recently, the technique/tool developed in this paper was successfully applied to a military case study [6]. Alloy was used to check and reason a plan ontology [12] developed by a research team at DSO National Laboratories in Singapore.

Recently, some researchers have begun to explore the potential of combining Web technologies and SE technologies together, e,g. [13]. However there has not been much work done on the application of formal techniques for semantic-web. In our previous work [5] we tried to extract web ontology from $\mathrm{Z}$ requirement models, which is a very different approach from the techniques demonstrated in this paper - checking and reasoning web ontology by encoding the semantics of DAML+OIL into the Alloy system.

From a completely different direction, i.e., applying SW to build software modeling environment, we recently investigated how RDF and DAML+OIL can be used to construct a Semantic Web environment for supporting, extending and 
integrating various specification languages [4]. We believe SW can contribute to the new developments for the software modeling environment.

In summary, there is a clear synergy between SW languages and software modeling techniques. The investigation of the links between those two paradigms will lead to great benefits for both areas.

\section{Acknowledgements}

We would like to thank Hugh Anderson, DSTA staffs and anonymous referees for many helpful comments. We also would thank Daniel Jackson and Ilya Shlyakhter for providing useful info and demo on Alloy. This work is supported by the Defence Innovative Research grant Formal Design Methods and DAML from Defence Science \& Technology Agency (DSTA) Singapore.

\section{References}

1. T. Berners-Lee, J. Hendler, and O. Lassila. The semantic web. Scientific American, May 2001.

2. D. Brickley and R.V. Guha (editors). Resource description framework (rdf) schema specification 1.0. http://www.w3.org/TR/2000/CR-rdf-schema20000327/, March, 2000.

3. J. Broekstra, M. Klein, S. Decker, D. Fensel, and I. Horrocks. Adding formal semantics to the web: building on top of rdf schema. In ECDL Workshop on the Semantic Web: Models, Architectures and Management, 2000.

4. J. S. Dong, J. Sun, and H. Wang. Semantic Web for Extending and Linking Formalisms. In L.-H. Eriksson and P. A. Lindsay, editors, Proceedings of Formal Methods Europe: FME'02, Copenhagen, Denmark, July 2002. Springer-Verlag.

5. J. S. Dong, J. Sun, and H. Wang. Z Approach to Semantic Web Services. In C. George and H. Miao, editors, International Conference on Formal Engineering Methods (ICFEM'02). LNCS, Springer-Verlag, October 2002.

6. J. S. Dong, J. Sun, H. Wang, C. H. Lee, and H. B. Lee. Analysing web ontology in alloy: A military case study. In Proc. 15th International Conference on Software Engineering and Knowledge Engineering: SEKE'03, San Francisco, USA, July 2003.

7. Richard Fikes and Deborah L. McGuinness. An axiomatic semantics for rdf, rdf schema, and daml+oil. Technical Report KSL-01-01, Knowledge Systems Laboratory, 2001.

8. I. Horrocks. The FaCT system. Tableaux'98, Lecture Notes in Computer Science, 1397:307-312, 1998.

9. D. Jackson. Micromodels of software: Lightweight modelling and analysis with alloy. Available: http://sdg.lcs.mit.edu/alloy/book.pdf, 2002.

10. D. Jackson, I. Schechter, and I. Shlyakhter. Alcoa: the alloy constraint analyzer. In Proc. 22nd International Conference on Software Engineering: ICSE'2000, pages 730-733, Limerick, Ireland, June 2000. ACM Press.

11. O. Lassila and R. R. Swick (editors). Resource description framework (rdf) model and syntax specification. http://www.w3.org/TR/1999/REC-rdf-syntax19990222/, Feb, 1999. 
12. C. H. Lee. Phase I Report for Plan Ontology. DSO National Labs, Singapore, 2002.

13. Cecilia Mascolo, Wolfgang Emmerich, and Anthony Finkelstein. XML technologies and software engineering. In International Conference on Software Engineering, pages 775-776, 2001.

14. F. van Harmelen, P. F. Patel-Schneider, and I. Horrocks (editors). Reference description of the daml+oil ontology markup language. Contributors: T. BernersLee, D. Brickley, D. Connolly, M. Dean, S. Decker, P. Hayes, J. Heflin, J. Hendler, O. Lassila, D. McGuinness, L. A. Stein, ..., March, 2001.

15. World Wide Web Consortium (W3C). Xsl transformations (xslt) version 1.0. http://www.w3.org/TR/xslt, 1999.

\section{A Completed DAML+OIL Semantic Encoding}

\section{A.1 Basic concepts}

The semantic models for DAML+OIL are encoded in the module DAMLOIL. The semantic encoding for the basic concepts was summarized in the table 2.

module DAMLOIL

Table 2. DAML+OIL Semantic encoding (basic concepts)

\begin{tabular}{||c|l||}
\hline DAML+OIL primitive & Alloy semantic function \\
\hline Resource & sig Resource \{\} \\
\hline$D A M L \_$Property & $\begin{array}{c}\text { disj sig Property extends Resource } \\
\text { sub_val: Resource } \rightarrow \text { Resource }\}\end{array}$ \\
\hline DAML_Class & $\begin{array}{c}\text { disj sig Class extends Resource } \\
\{\text { instances: set Resource }\}\end{array}$ \\
\hline Datatype & disj sig Datatype extends Class \{\} \\
\hline
\end{tabular}

All the things described in Semantic web context are called resources. All other concepts defined later like Property and Class are extended from the Resource.

\section{A.2 Class Elements}

The semantic encoding for the class elements was summarized in the table 3 . It includes constructs like subClassOf, disjointWith, disjointUnionOf and sameClassAs.

\section{A.3 Property Restrictions}

The semantic encoding for the property restrictions was summarized in the table 4. A property restriction defines the class of all objects that satisfy the restriction. For example the toClass function states that all instances of the class $\mathrm{c} 1$ have the values of property $P$ all belonging to the class $\mathrm{c} 2$. The other constructs include hasValue, hasClass, cardinality etc.. 
Table 3. DAML+OIL Semantic encoding (class elements)

\begin{tabular}{||c|c||}
\hline DAML+OIL primitive & Alloy semantic function \\
\hline subClassOf & $\begin{array}{c}\text { fun subClassOf(csup, csub: Class) } \\
\text { \{csub.instances in csup.instances }\}\end{array}$ \\
\hline disjointWith & fun disjointWith (c1, c2: Class) \\
& $\{$ no c1.instances \& c2.instances $\}$ \\
\hline disjointUnionOf & fun disjointUnionOf(clist: List, c1: Class) \\
& $\{$ c1.instances $=$ clist.*next.val.instances \\
& all disj ca1, ca2: clist.*next.val \\
& no ca1.instances \& ca2.instances $\}$ \\
\hline sameClassAs & fun sameClassAs( c1, c2: Class) \\
& $\{$ c1.instances $=$ c2.instances $\}$ \\
\hline
\end{tabular}

Table 4. DAML+OIL Semantic encoding (Property restrictions)

\begin{tabular}{|c|c|}
\hline DAML+OIL primitive & Alloy semantic function \\
\hline toClass & $\begin{array}{l}\text { fun toClass (p: Property, c1: Class, c2: Class) } \\
\{\text { all r1, r2: Resource | r1 in c1.instances }<=> \\
\text { r2 in r1. }(\text { p.sub_val })=>\text { r2 in c2.instances }\}\end{array}$ \\
\hline hasValue & $\begin{array}{l}\text { fun hasValue (p: Property, c1: Class, } \mathrm{r} \text { : Resource) } \\
\{\text { all } \mathrm{r} 1 \text { : Resource | r1 in c1.instances }=> \\
\text { r1.(p.sub_val)=r }\}\end{array}$ \\
\hline hasClass & $\begin{array}{l}\text { fun hasClass (p: Property, c1: Class, c2: Class) } \\
\text { \{all r1: Resource | r1 in c1.instances => } \\
\text { some r1.(p.sub_val) \& c2.instances }\}\end{array}$ \\
\hline cardinality & $\begin{array}{l}\text { fun cardinality (p: Property, c1: Class, N: Int) } \\
\{\text { all r1: Resource | r1 in c1.instances }<=> \\
\quad \# \text { r1. }(\text { p.sub_val })=\text { int } \mathrm{N}\}\end{array}$ \\
\hline maxCardinality & $\begin{array}{l}\text { fun maxCardinality (p: Property, c1: Class, N: Int) } \\
\{\text { all r1: Resource | r1 in c1.instances }<=> \\
\text { \# r1.(p.sub_val) }=<\text { int } \mathrm{N}\}\end{array}$ \\
\hline minCardinality & $\begin{array}{c}\text { fun minCardinality (p: Property, c1: Class, N: Int) } \\
\{\text { all r1: Resource | r1 in c1.instances }<=> \\
\# \text { r1.(p.sub_val) }>=\text { int } \mathrm{N}\}\end{array}$ \\
\hline cardinality $Q$ & $\begin{array}{c}\text { fun cardinalityQ (p: Property, c1: Class, } \mathrm{N} \text { : Int, c2: Class) } \\
\{\text { all r1: Resource | r1 in c1.instances }<=> \\
\# \text { r1.(p.sub_val) \& c2.instances }=\text { int } \mathrm{N}\}\end{array}$ \\
\hline $\max C a r d i n a l i t y Q$ & $\begin{array}{l}\text { fun maxCardinalityQ (p: Property, c1: Class, N: Int, c2: } \\
\text { Class) } \\
\quad\{\text { all r1: Resource | r1 in c1.instances }<=> \\
\quad \# \text { r1.(p.sub_val) \& c2.instances }=<\text { int } \mathrm{N}\}\end{array}$ \\
\hline minCardinality $Q$ & $\begin{array}{l}\text { fun minCardinalityQ(p: Property, c1: Class, N: Int, c2: } \\
\text { Class) } \\
\text { \{all r1: Resource | r1 in c1.instances }<=> \\
\quad \# \text { r1.(p.sub_val) \& c2.instances }>=\text { int } \mathrm{N}\}\end{array}$ \\
\hline
\end{tabular}




\section{A.4 Boolean Combination of Class Expressions}

The semantic encoding for the boolean combination of class expression was summarized in the table 5 .

Table 5. DAML+OIL Semantic encoding (Boolean combination)

\begin{tabular}{|c|c|}
\hline DAML+OIL primitive & Alloy semantic function \\
\hline intersectionOf & $\begin{array}{l}\text { fun intersectionOf (clist: List, c1: Class) } \\
\text { \{all } \mathrm{r} \text { : Resource } \mathrm{r} \text { in } \mathrm{c} 1 . \text { instances }<=> \\
\left.\quad \text { all ca: clist. }{ }^{*} \text { next.val } \mid \mathrm{r} \text { in ca.instances }\right\}\end{array}$ \\
\hline unionOf & $\begin{array}{l}\text { fun unionOf (clist: List, c1: Class) } \\
\quad \text { all } \mathrm{r} \text { : Resource } \mathrm{r} \text { in } \mathrm{c} 1 . \text { instances }<=> \\
\quad \text { some ca: clist.*next.val } \mid \mathrm{r} \text { in ca.instances }\}\end{array}$ \\
\hline
\end{tabular}

\section{A.5 Property Elements}

The semantic encoding for the property elements was summarized in the table 6 . It includes subPropertyOf, samePropertyAs etc..

Table 6. DAML+OIL Semantic encoding (Property elements)

\begin{tabular}{|c|c|}
\hline DAML+OIL primitive & Alloy semantic function \\
\hline subPropertyOf & $\begin{array}{c}\text { fun subPropertyOf (psup, psub: Property) } \\
\text { \{psub.sub_val in psup.sub_val }\}\end{array}$ \\
\hline domain & $\begin{array}{l}\text { fun domain (p: Property, c: Class) } \\
\{\text { (p.sub_val).Resource in c.instances }\}\end{array}$ \\
\hline range & $\begin{array}{l}\text { fun range (p: Property, c: Class) } \\
\quad\{\text { Resource.(p.sub_val) in c.instances }\}\end{array}$ \\
\hline samePropertyAs & $\begin{array}{c}\text { fun samePropertyAs(p1, p2: Property) } \\
\{\text { p1.sub_val=p2.sub_val }\}\end{array}$ \\
\hline inverseOf & $\begin{array}{l}\text { fun inverseOf }(\mathrm{p} 1, \mathrm{p} 2: \text { Property }) \\
\{\mathrm{p} 1 . \text { sub_val }=\tilde{(\mathrm{p} 2 . \text { sub_val })\}}\end{array}$ \\
\hline TransitiveProperty & $\begin{array}{l}\text { fun TransitiveProperty(p: Property) } \\
\text { \{all x, y, z: Resource | } \\
\text { y in (p.sub_val).x \&\& z in (p.sub_val).y => } \\
\text { z in (p.sub_val).x }\}\end{array}$ \\
\hline UniqueProperty & $\begin{array}{l}\text { fun UniqueProperty (p: Property) } \\
\quad\{\text { all x : Resource | sole x.(p.sub_val) }\}\end{array}$ \\
\hline UnambigousProperty & $\begin{array}{l}\text { fun UnambigousProperty(p: Property) } \\
\quad\{\text { all } \mathrm{x} \text { : Resource | sole (p.sub_val).x }\}\end{array}$ \\
\hline
\end{tabular}

